\title{
Optical Properties of Carbonaceous Aerosol Particles by Low-Loss EELS
}

\author{
D.T.L. Alexander,* P.A. Crozier,* and J. Anderson** \\ * Center for Solid State Science, Arizona State University, PO Box 871704, Tempe, AZ 85282 \\ ** Department of Mechanical \& Aerospace Engineering, Arizona State University, PO Box 876106, \\ Tempe, AZ 85282
}

A detailed understanding of the physical properties of pollutant carbonaceous aerosol particles, such as diesel or coal soot, is essential for accurate predictive modeling of climate change resulting from anthropogenic activities. The optical properties of such particles, referred to as black carbon (BC), are particularly important, since they determine light scattering and absorption and, thus, the radiative balance of the aerosol. A key determinant of optical properties is the dielectric response function of a material. In this work, we measure the dielectric functions of individual carbonaceous aerosol particles over the visible wavelengths using electron energy-loss spectroscopy (EELS) in the transmission electron microscope (TEM). The method behind this is to transform the low-loss EEL spectrum into plots of the real and imaginary dielectric constants against energy loss using the Kramers-Kronig procedure. Then, ultimately, it should be possible to convert these into the real and imaginary refractive indices for particles.

The part of the low-loss spectrum that corresponds to visible wavelengths is between about 1 to 4 $\mathrm{eV}$. Until recently, making accurate energy-loss measurements at such low losses was very difficult, because of the significant background from the zero-loss peak. However, the recent availability of monochromated electron guns greatly increases the possibility of studying features in the visible region of the spectrum. We have recorded EEL spectra from BC particles of climatically-important Asian Pacific samples in a monochromated FEI Tecnai F20 TEM [1]. The instrument was operated with a beam voltage of $200 \mathrm{kV}$ and using an objective aperture to limit the collection semi-angle to 8 mrad. To solve the problem of detector saturation from the intense zero-loss signal limiting the intensity recorded from the low-loss region, we use the approach in which a spectrum of short acquisition time that includes the zero-loss peak is spliced to one of longer acquisition time with the zero-loss peak shifted off the spectrometer [2].

Figs. 1 and 2 show some results. In Fig. 1, there are examples of two of the typical types of BC particles found in the samples. They have different morphologies and structure, owing to different formation conditions. Fig. 2 shows the corresponding low-loss spectra, with zero-loss removed and deconvolved to give single-scattering distributions. Preliminary calculations of the dielectric response functions against energy-loss have then been made, also in Fig. 2. Although these calculations and the determination of the inelastic and then single-scattering distributions from the initial data are all subject to further refinements, already important conclusions can be drawn. Firstly, the low-loss EEL spectra for the two particles are very different. Secondly, this variance is manifested in the dielectric functions, which are very different to each other in the critical optical regime of 1 to $4 \mathrm{eV}$.

Currently, the work raises significant questions as to the relationship of the low energy-loss spectrum with both the dielectric constants of small particles and also the optical properties of those particles. Satisfactory answers to these questions could not only render these results useful to the 
understanding of how aerosols affect climate, but also bring this methodology to the difficult question of determining optical properties of small particles in general, for instance helping determine how the optical properties of small particles relate to the bulk optical properties.

\section{References}

[1] A.D. Clarke et al., J. Geophys. Res. - Atmos. 109 (2004) D15S09.

[2] R. Erni and N.D. Browning, Ultramic. 104 (2005) 176.

[3] This work was supported by NASA grant NNG04GB37G. The authors acknowledge support of the National Center for Electron Microscopy, Lawrence Berkeley Lab, which is supported by the U.S. Department of Energy under Contract \# DE-AC02-05CH11231.
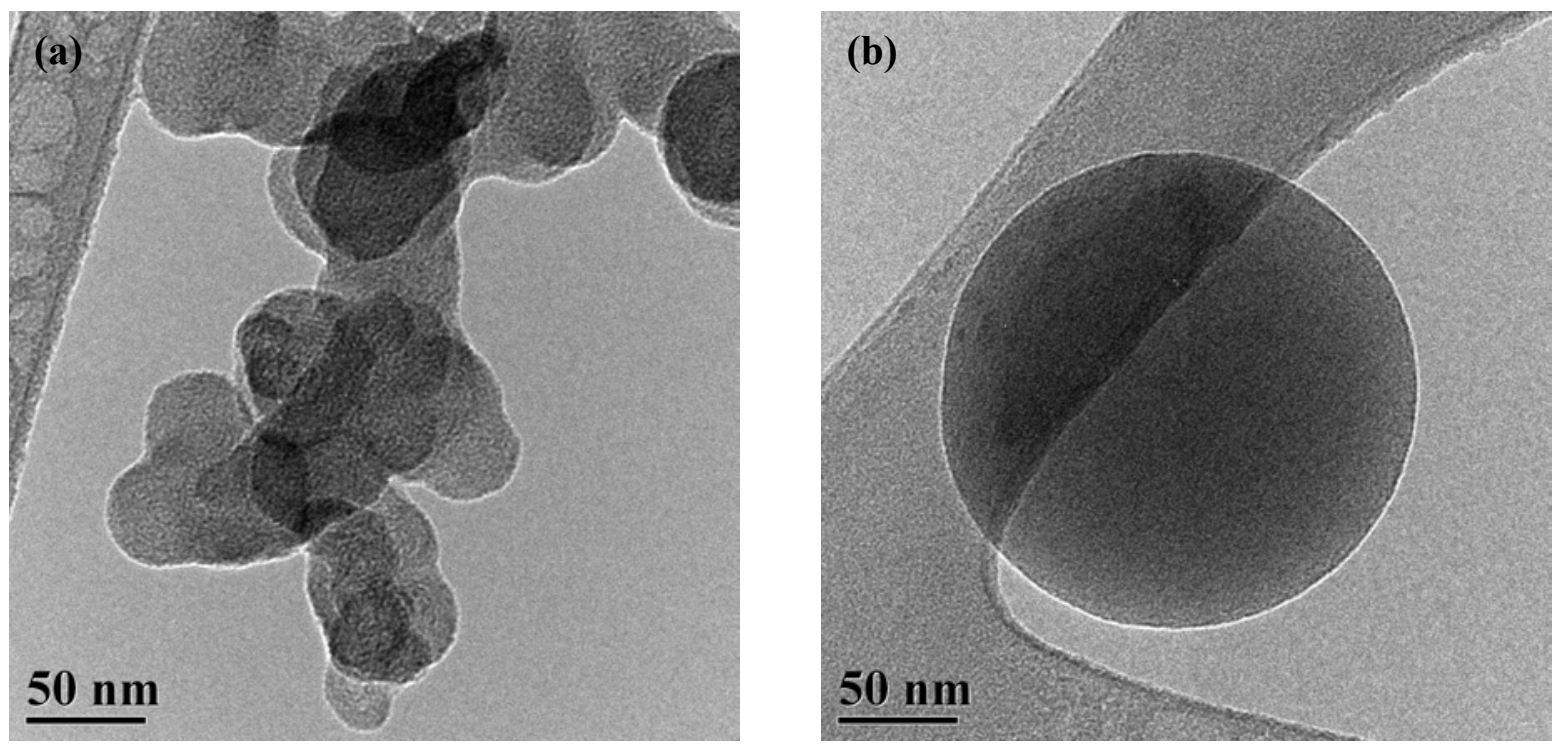

FIG. 1. Two typical types of carbonaceous particle found in the Asian Pacific samples. First, a sooty chain consisting of an aggregate of small carbon spherules with a somewhat graphitic structure, (a), and second an isolated, amorphous carbon sphere, (b).
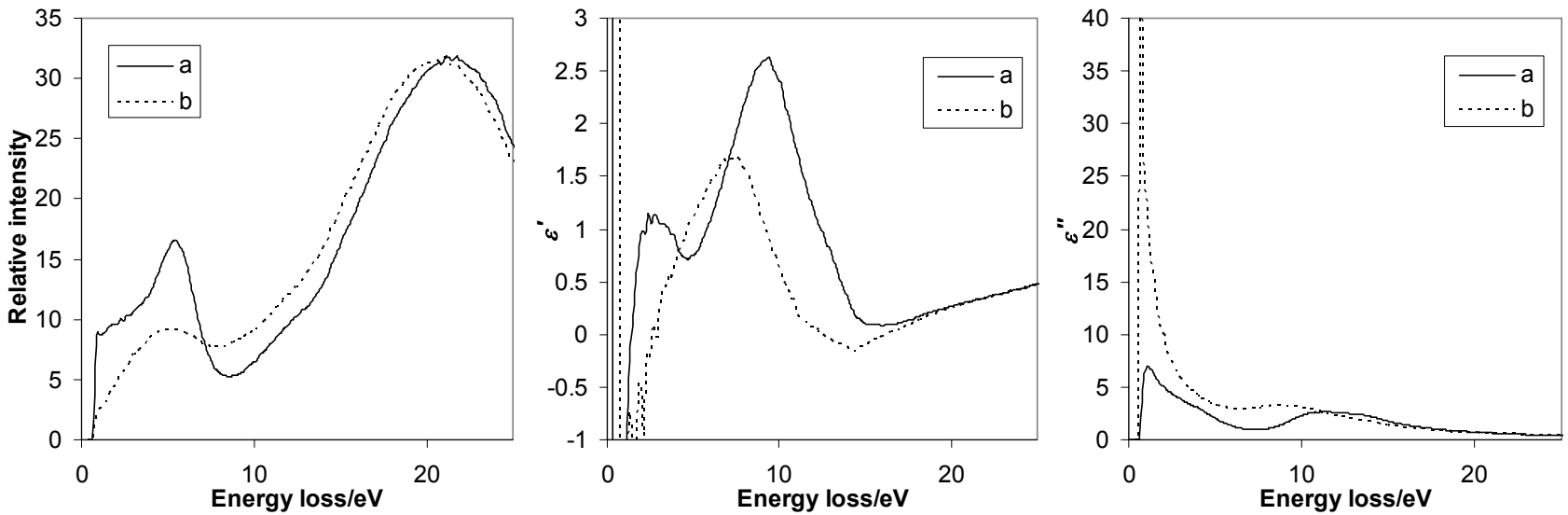

FIG. 2. The left figure shows the single-scattering EEL spectra for particles (a) and (b) illustrated in Fig. 1. From these, the real and imaginary dielectric constants, $\varepsilon$ ' and $\varepsilon$ ', have been calculated, as shown on the middle and right figures. The particles clearly have different dielectric response functions between 1 to $4 \mathrm{eV}$ energy loss, which corresponds to the optical regime. 